\title{
ON BOUNDS FOR DISCRETE SEMIGROUPS
}

\author{
KreŠIMIR VeSElić AND NinOSlaV TRUhaR
}

Abstract. The main result of this note is extension on the infinite dimension of the following known result for finite matrices: while the spectral radius $\rho(T)$ gives only asymptotic decay estimates, the solution $X$ of the discrete Lyapunov equation $X-T^{*} X T=B B^{*}$ yields rigorous bounds. We also present a new upper bound for the norm of the solution $X$ in the matrix case which depends on the structure of the right hand side. The new bound shows that the structure of $B$ can greatly influence $\|X\|$.

Mathematics subject classification (2000): 11D04, 11D61, 15A24, 15A90.

Keywords and phrases: Exponential decay, discrete Lyapunov equation, upper bounds.

\section{REFERENCES}

[1] B.N. DatTA, Numerical Methods for Linear Control Systems, Elsevier Academic Press, London, UK, 2004.

[2] H. Dym, Linear Algebra in Action, Graduate Studies in Mathematics 78, Amer. Math. Soc., Providence RI, 2006.

[3] S. K. Godunov, Modern Aspects of Linear Algebra, Translations of Mathematical Monographs, Amer. Math. Soc. Providence, RI, Vol. 175, 1998.

[4] I. Gohberg, S. Gohberg And M. A. KaAshoek, Classes of Linear Operators, Vol.I, Birkhäuser OT 49, Basel and Boston, 1990.

[5] T. Kato, Perturbation Theory for Linear Operators, 2nd ed., Berlin Heidelberg New York 1980, 1995.

[6] N. Truhar, K. Veselić, Bounds on the trace of solution to the Lyapunov equation with a general stable matrix, Systems and Control Letters, 56 (2007), 493-503. 